REVISTA CIENCIAS BIOMÉDICAS

ARTÍCULOS DE REVISIÓN

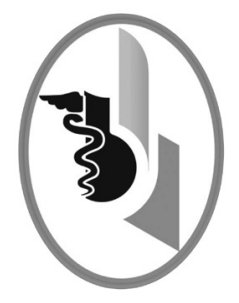

\title{
VENTAJAS DE LA COLECISTECTOMÍA TRANSVAGINAL
}

ADVANTAGES OF THE TRANSVAGINAL CHOLECYSTECTOMY

\author{
Arcila-Olmos Viviana ${ }^{1}$ \\ Pineda-Quintero Alejandra J.2 \\ Sánchez-Bustamante Luisa F. ${ }^{2}$ \\ Correspondencia: luisancheb@gmail.com \\ Recibido para evaluación: enero-5-2015. Aceptado para publicación: mayo-29-2015.
}

\section{RESUMEN}

Introducción: acceder por orificios naturales para realizar procedimientos, es un avance en las técnicas quirúrgicas. Estos abordajes tienen múltiples ventajas estéticas, menor riesgo de complicaciones y menor tiempo postoperatorio. Es por eso que abordar la vesícula biliar por vía transvaginal, surge como estrategia para el manejo de patologías biliares.

Objetivo: identificar las ventajas que tiene la colecistectomía transvaginal (CTV), como método novedoso y menos invasivo para el tratamiento de la litiasis biliar.

Método: se efectuó revisión en las bases Pubmed, Scielo y Google académico, con selección de artículos publicados entre el año 2007-2013.

Resultados: se encontraron 18 artículos. Fueron revisados en texto completo y seis $(33.0 \%)$ se consideraron adecuados para la revisión. Las seis series de pacientes suman en total de 375 mujeres, intervenidas por CTV debido a la presencia de colelitiasis o colecistitis. Las características generales observadas fueron: [A] Edad promedio: 42.2 años. [B] ASA: entre I y II. [C] IMC promedio: $27.6 \mathrm{~kg} / \mathrm{m} 2$. [D] Tiempo quirúrgico promedio: 72.3 minutos. Se detallaron las complicaciones intraoperatorias y postoperatorias señaladas en los estudios.

Conclusión: la CTV ofrece beneficios en cuanto a estética y menor necesidad de analgesia, no obstante es mayor el tiempo postoperatorio. Aún no se tiene suficientes estudios en CTV que demuestren las ventajas en cuanto a complicaciones a corto plazo, como dispareunia o a largo plazo como problemas en fertilidad. Rev.cienc.biomed. 2015;6(1):130-137

\section{PALABRAS CLAVE}

Colecistectomía; Colelitiasis; Colecistectomía Laparoscópica; Cirugía Endoscópica por Orificio Natural

\section{SUMMARY}

Introduction: to gain access by natural holes, to do procedures, is an advance that has appeared in the surgical techniques. These approaches have multiple advantages, between them, the esthetic, less complications and lower postoperative time. It is because of it that to gain access to the gallbladder by transvaginal way appears as strategy for the management of biliary tract pathologies.

Instrumentadora Quirúrgica. Magíster en Epidemiología. Docente. Facultad de Medicina. Universidad de Antioquia. Colombia. Instrumentadora Quirúrgica. Facultad de Medicina. Universidad de Antioquia. Colombia 
Objective: to identify in published articles, the advantages that have the transvaginal cholecystectomy (TVC), as novel and less invasive method for the treatment of cholelithiasis

Methods: review of databases Pubmed, Scielo and academic google, with selection of articles published since 2007 to 2013.

Results: 18 articles were found. They were reviewed in full text and six $(33.0 \%)$ were considered to convenience adequate for the review. The six series of patients made a total of 375 women, operated by TVC due to the presence of cholelithiasis or cholecystitis. The general characteristics observed were: [A] Average age: 42.2 years. [B] ASA: between I and II. [C] Average BMI: $27.6 \mathrm{Kg} / \mathrm{m} 2$. [D] Average time of the surgical procedure: 72.3 minutes. The intraoperative and postoperative complications indicated in the studies were detailed.

Conclusions: the TVC offers benefits with regard to the esthetics and less necessity of analgesics, however the postoperative time is higher. Still, there are not sufficient studies in TVC that strongly demonstrate the advantages with regards to the short-term complications as dyspareunia or to long-time as fertility problems. Rev.cienc.biomed. 2015;6(1):130-137

\section{KEYWORDS}

Cholecystectomy; Cholelithiasis; Laparoscopic cholecystectomy; Natural Orifice

Endoscopic Surgery.

\section{INTRODUCCIÓN}

La vesícula biliar es un saco muscular membranoso de reserva, en la cual se almacena la bilis hepática. Tiene una estrecha relación anatómica con el hígado, está situada por debajo de este, en un sitio conocido como lecho hepático (1). Su función principal es servir de depósito de la bilis hepática, facilitando la digestión y la consiguiente absorción de los alimentos. La vesícula biliar se comunica con el tracto gastrointestinal a través del conducto colédoco, este se inserta en la segunda porción del duodeno, en lo que se conoce como Ampolla de Váter, la cual consta de un esfínter que controla la inducción de la bilis y de los jugos pancreáticos al duodeno y previene el reflujo o entrada retrógrada del contenido duodenal por la ampolla, este esfínter se conoce como esfínter de Oddi (1).

La principal causa de enfermedad biliar es la formación de cálculos, seguida por la formación de pólipos, que según estudios realizados en Chile tienen una frecuencia de $3.1 \%$, con una relación hombre:mujer de $1: 1.1$; siendo más usuales los pólipos de colesterol (67.9\%) y los hiperplásicos (20.3\%) (2).

Otra de las patologías de la vía biliar es el cáncer, que es poco frecuente en los países desarrollados con una tasa de incidencia de 1.3 casos por 100.000 habitantes en mujeres y de 0.8 casos por 100.000 en hombres; mientras que en las poblaciones de alto riesgo, como los países andinos, se reportan tasas de incidencia de $10-15$ casos por 100.000 habitantes en mujeres y de 3.5-7.0 casos en hombres, relacionando estos resultados con las características genéticas, hormonales y ambientales (3).

La indicación quirúrgica más común para extraer la vesícula biliar es la colelitiasis. Una de las enfermedades con mayor incidencia en la población adulta, la cual varía de una zona geográfica a otra. En la población estadounidense, se calcula que entre el 10$15 \%$ de los adultos padecen de colelitiasis y cada año se diagnostican aproximadamente 800.000 casos nuevos. Asimismo, en América Latina, especialmente en Chile y Argentina, se calculan tasas similares a las de Norte América. Se afirma que tanto la incidencia como la prevalencia son mayores en el sexo femenino con una relación de $3: 1$ y que dicha tendencia aumenta con la edad, especialmente después de los 40 años, donde el $20 \%$ de los habitantes de occidente padecen de cálculos, el $25 \%$ después de los 60 años y el $33 \%$ después de los 70 . Se observan, sin embargo, cálculos en adolescentes y muy raramente en niños menores de 10 años (4).

En América Latina, estudios han demostrado que entre el $5-15 \%$ de los habitantes presentan litiasis biliar y existen poblaciones y/o etnias con mayores prevalencias como 
la hispánica, la caucásica y los nativos americanos (5). Es por esto que la litiasis de la vesícula biliar o colelitiasis, se ha considerado una de las patologías más relevantes y antiguas que han afectado al ser humano.

Hoy se conoce que el vaciamiento del contenido vesícular se deteriora con el envejecimiento y tiene relación con la sintomatología, siendo la edad el principal factor de riesgo (6). En segundo lugar, el sexo femenino ha sido más propenso a formar cálculos en la vesícula biliar, pues la patología también se relaciona con el embarazo, la paridad, el tratamiento con anticonceptivos y la terapia de reemplazo con estrógenos en la menopausia, además los niveles elevados de estrógenos séricos activan la secreción de bilis sobresaturada de colesterol y los elevados niveles de progesterona que causan éxtasis vesicular $(5,6)$.

Es una enfermedad multifactorial cuya patogénesis no se conoce con precisión, a veces, se asocia con historia familiar, diabetes mellitus, obesidad, pérdida masiva de peso y enfermedades hemolíticas (7). Es una patología que puede generar cuadros clínicos muy complejos y dolorosos para el paciente. Sin embargo, en ocasiones, es difícil crear un estudio claro de prevalencia e incidencia de la enfermedad, porque entre un $65-80 \%$ de la litiasis de la vesícula biliar no produce síntomas (8). Su diagnóstico se da en muchas ocasiones de manera casual en exploraciones ecográficas. El cólico biliar es la manifestación clínica más común.

Debido a las múltiples complicaciones que la patología puede presentar y su frecuencia de aparición, se ha establecido el tratamiento quirúrgico, desde que Lagenbuchen 1881, realizara la primera colecistectomía con éxito. De allí, que la colecistectomía se haya convertido en uno de los actos quirúrgicos abdominales más habituales y es uno de los procedimientos con más equilibrio costo-beneficio en la actualidad (9).

Tanto fue el avance científico para el tratamiento de los cálculos biliares que en 1987 Mourety Dubois en Francia y en 1988, Mc Kernany Reddick en los Estados Unidos, introdujeron el abordaje laparoscópico (10), con el fin de reducir el tamaño de la incisión, el tiempo quirúrgico y la necesidad de analgesia durante el postoperatorios, además de disminuir el índice de morbimortalidad.

La cirugía laparoscópica permitió cambios técnicos en el manejo de la patología. Los cirujanos que la practican hablan en términos de mínimo acceso, ameritaron adquirir nuevas habilidades para la introducción de equipos e instrumentos de tecnología más avanzada y consiguieron mejor visualización del campo operatorio (11). Más recientemente se propone la minilaparoscopia con instrumental de menor diámetro del que utiliza la laparoscopia convencional, requiriendo incisiones de menos de un centímetro (12).

No obstante, el abordaje laparoscópico no fue una solución final, hoy aparecen nuevos intentos de acceso a la zona quirúrgica biliar, sin dejar huella en la piel abdominal. Uno de los conceptos más novedosos es la cirugía endoscópica transluminal a través de orificios naturales [NOTES], una técnica implementada primero en animales y que se inicia su propuesta en humanos (12). En esta técnica, los instrumentos se introducen en la cavidad peritoneal a través de la vagina, vejiga, estómago, o recto. El abordaje transvaginal ha ganado más popularidad, evita las cicatrices visibles, aunque requiere instrumentos muy flexibles y de mayor longitud para poder acceder al sitio quirúrgico.

La "cirugía sin huella" teóricamente, reduce el trauma quirúrgico y podría mejorar los resultados clínicos con la reducción del dolor postoperatorio y una recuperación más rápida; además de lograr excelente resultado cosmético. En el desarrollo de la cirugía transvaginal, el interés se ha centrado en las complicaciones potenciales, tanto en las relacionadas con la colecistectomía, como en la potencial infección ginecológica. Teniendo en cuenta lo anterior, el objetivo del estudio fue buscar en las publicaciones científicas las ventajas de la CTV en el tratamiento de la litiasis biliar.

\section{MATERIAL Y MÉTODOS}

Se efectuó revisión de tema sobre la práctica de CTV. Se condensaron los hallazgos y las 
conclusiones de los diferentes artículos con el fin de comprender mejor los resultados encontrados.

Los pasos que se realizaron para la elaboración de esta revisión fueron los siguientes: selección del tema a estudiar, formulación de la pregunta de investigación, estrategias de búsqueda empleadas, definición de los criterios de inclusión y exclusión, selección de los estudios a analizar, interpretación de los resultados y elaboración del artículo.

La pregunta formulada para la investigación fue: ¿Qué ventajas ofrece la CTV como nuevo tratamiento quirúrgico? Se incluyeron los artículos que contenían información acerca de la realización de CTV en humanos, delimitándolos a los idiomas de inglés y español. Se escogieron artículos publicados entre el año 2007 - 2013.

La investigación fue realizada en la base de datos MEDLINE y LILACS, apoyándonos en fuentes científicas y médicas como Scielo y Pubmed con las siguientes palabras clave: colecistectomía transvaginal, NOTES [Natural orífices Trasluminal Endoscopic Surgery] y colecistectomía calidoscópica.

Primero se seleccionaron los artículos que cumplían con los criterios de inclusión del estudio, básicamente CTV. Fueron leídos y analizados en su totalidad. Se consideraron: año de publicación, número de pacientes intervenidos, edad de las pacientes, procedimientos abdominales previos, riesgo anestésico, tiempo quirúrgico, complicaciones intraoperatorias y postoperatorias, dolor postoperatorio y estancia hospitalaria.

\section{RESULTADOS}

Se encontraron 18 artículos en total, 15 provenientes de la base de datos MEDLINE y tres de LILACS. Se descartaron 12 después de hacer un análisis crítico de cada uno. Finalmente la muestra de estudio seleccionada quedó con los seis artículos que cumplían con los criterios de inclusión del estudio. De estos, cinco eran estudios prospectivos (1317) y uno un análisis comparativo de la CTV con la laparoscópica convencional (18).
En relación al origen de las publicaciones, se identificó un mayor número en Europa, de la que hacen parte cinco artículos, siendo dos de Alemania $(15,18)$, dos de España $(13,17)$ y uno de los Países Bajos (14). El sexto artículo era procedente de los Estados Unidos (16). Estos artículos se sitúaban entre los años 2010 - 2013 (Tabla N¹).

En los estudios se suman 375 pacientes, de género femenino, que se intervinieron por la presencia de colelitiasis o colecistitis mediante la técnica TVC. Las características generales de los pacientes en cuanto a edad fueron: promedio de 42.2 años de edad y el IMC promedio de $27.6 \mathrm{Kg} / \mathrm{m}^{2}$. Tabla $\mathrm{N}^{\circ} 2$. La clasificación del riesgo anestésico de la ASA fue de I y II en todos los estudios.

Tabla No 2.

EDAD E ÍNDICE DE MASA CORPORAL (IMC)

\begin{tabular}{|l|c|c|}
\hline \multicolumn{1}{|c|}{ Autor } & $\begin{array}{c}\text { Promedio de } \\
\text { edades, } \\
\text { Años }\end{array}$ & $\begin{array}{c}\text { Promedio } \\
\text { de IMC, } \\
\mathrm{Kg} / \mathrm{m}^{2}\end{array}$ \\
\hline Nijhawan (16) & 40.1 & 25.2 \\
\hline Cuadrado (13) & 39.7 & 35.0 \\
\hline Van de Boezem (14) & 38.0 & 25.0 \\
\hline Noguera (17) & 39.7 & $=$ \\
\hline H. Mofid (15) & 47.0 & 27.0 \\
\hline Zornig (18) & 49.0 & 26.0 \\
\hline
\end{tabular}

El tiempo quirúrgico promedio fue de 72.3 minutos con un rango de 23-165. Las intervenciones iniciales se asociaron con tiempos quirúrgicos más largos debido a la curva de aprendizaje. La estancia hospitalaria varió en los diferentes estudios, desde horas a días, aunque la mayoría fueron dados de alta el primer día del postoperatorio. Las pacientes en quiénes se tuvo que convertir el procedimiento a otra técnica, la estancia hospitalaria fue mayor (Tabla $\mathrm{N}^{\circ} 3$ ).

En cuanto al dolor postoperatorio, en el estudio comparativo se observó que requirieron menos narcóticos en la unidad de recuperación postanestésica las intervenidas por CTV (una) que convencional (ocho). Los niveles de dolor fueron más bajos para el grupo de CTV en los días primero, cuarto y séptimo postoperatorio. 
TABLA $\mathbf{N}^{\circ} 1$ ESTUDIOS SELECCIONADOS

\begin{tabular}{|c|c|c|c|c|c|}
\hline Autores & Año & Tipo & País & Muestra & Periodo \\
\hline $\begin{array}{l}\text { Nijhawan et al. } \\
\text { (16) }\end{array}$ & 2012 & $\begin{array}{l}\text { Estudio } \\
\text { prospectivo }\end{array}$ & $\begin{array}{l}\text { Estados } \\
\text { Unidos }\end{array}$ & $\begin{array}{l}\text { Hospital: } 27 \text { mujeres } \\
\text { con diagnóstico } \\
\text { de enfermedad } \\
\text { de la vesícula } \\
\text { biliar, sometidos } \\
\text { a colecitectomia } \\
\text { transvaginal híbrida }\end{array}$ & $\begin{array}{l}\text { Septiembre } \\
2007 \\
\text { Abril } \\
2011\end{array}$ \\
\hline $\begin{array}{l}\text { Cuadrado et al. } \\
\text { (13) }\end{array}$ & 2010 & $\begin{array}{l}\text { Estudio } \\
\text { prospectivo }\end{array}$ & España & $\begin{array}{l}\text { Hospital: } 25 \\
\text { mujeres sometidas } \\
\text { a colecistectomía } \\
\text { transvaginal híbrida } \\
\text { por colelitiasis no } \\
\text { complicada. }\end{array}$ & 140 días \\
\hline $\begin{array}{l}\text { Van den Boezem et } \\
\text { al. (14) }\end{array}$ & 2013 & $\begin{array}{l}\text { Estudio } \\
\text { prospectivo }\end{array}$ & Países Bajos & $\begin{array}{l}\text { Base de datos: } \\
50 \text { pacientes con } \\
\text { colelitiasis sintomática } \\
\text { a los que se le realizo } \\
\text { TVC. }\end{array}$ & $\begin{array}{l}\text { Enero } \\
2011 \\
\text { Abril } \\
2012\end{array}$ \\
\hline $\begin{array}{l}\text { Noguera et al. } \\
(17)\end{array}$ & 2009 & $\begin{array}{l}\text { Estudio } \\
\text { prospectivo } \\
\text { observacional }\end{array}$ & España & $\begin{array}{l}\text { Base de datos: } \\
15 \text { pacientes para } \\
\text { colecistectomía } \\
\text { electiva por } \\
\text { colecistolitiasis }\end{array}$ & $\begin{array}{l}\text { Octubre } \\
2008 \\
\text { Octubre } \\
2009\end{array}$ \\
\hline $\begin{array}{l}\text { H. Mofid et al. } \\
\text { (15) }\end{array}$ & 2011 & $\begin{array}{l}\text { Estudio } \\
\text { prospectivo }\end{array}$ & Alemania & $\begin{array}{l}\text { Hospital: } 158 \text { mujeres } \\
\text { sometidas a TVC, por } \\
\text { colelitiasis. }\end{array}$ & $\begin{array}{c}\text { Junio } \\
2007 \\
\text { Septiembre } \\
2010 \\
\end{array}$ \\
\hline $\begin{array}{l}\text { Zornig et al. } \\
(18)\end{array}$ & 2010 & $\begin{array}{l}\text { Estudio } \\
\text { comparativo. }\end{array}$ & Alemania & $\begin{array}{l}\text { Banco de datos: } \\
100 \text { pacientes con } \\
\text { inflamación aguda o } \\
\text { crónica de la vesícula } \\
\text { biliar, que fueron } \\
\text { sometidos a TVC }\end{array}$ & $\begin{array}{c}\text { Junio } \\
2007 \text { Febrero } \\
2009\end{array}$ \\
\hline
\end{tabular}

\begin{tabular}{|c|c|c|c|c|c|c|}
\hline \multicolumn{7}{|c|}{$\begin{array}{c}\text { TABLA N³. } \\
\text { INTERVENCIÓN OPERATORIA }\end{array}$} \\
\hline & $\begin{array}{l}\text { Nijhawan } \\
(16)\end{array}$ & $\begin{array}{c}\text { Cuadrado } \\
\text { (13) }\end{array}$ & $\begin{array}{l}\text { Van de Boezem } \\
(14)\end{array}$ & $\begin{array}{c}\text { Noguera } \\
(17)\end{array}$ & $\begin{array}{l}\text { H. Mofid } \\
(15)\end{array}$ & $\begin{array}{c}\text { Zornig } \\
(18)\end{array}$ \\
\hline $\begin{array}{l}\text { Tiempo } \\
\text { quirúrgico }\end{array}$ & 72 Min & 89,5 Min & $61 \mathrm{Min}$ & $86,62 \mathrm{Min}$ & 50 Min & 52 Min \\
\hline $\begin{array}{l}\text { Complicaciones } \\
\text { intraoperatorias }\end{array}$ & - & - & - & - & $\begin{array}{l}\text { Perforación de } \\
\text { la vejiga }\end{array}$ & - \\
\hline $\begin{array}{l}\text { Complicaciones } \\
\text { posoperatorias }\end{array}$ & - & $\begin{array}{l}\text { Hematuria } \\
\text { Infección } \\
\text { del tracto } \\
\text { urinario }\end{array}$ & $\begin{array}{l}\text { Infección } \\
\text { de herida } \\
\text { abdominal } \\
\text { Infección } \\
\text { fúngica vaginal }\end{array}$ & Hematuria & $\begin{array}{c}\text { Absceso en el } \\
\text { fondo de saco } \\
\text { de Douglas }\end{array}$ & $\begin{array}{c}\text { Absceso } \\
\text { en el fondo } \\
\text { de saco de } \\
\text { Douglas }\end{array}$ \\
\hline $\begin{array}{l}\text { Estancia } \\
\text { hospitalaria, } \\
\text { (Días) }\end{array}$ & $1-2$ & 1 & $0-1$ & $1-2$ & $1-7$ & $1-7$ \\
\hline
\end{tabular}


Las pacientes que habían tenido cirugía abdominal o pélvica previamente, fueron 125 $(33.3 \%)$, siendo prevalentes: apendicetomía, histerectomía, cesárea y oforectomía. Ello no fue impedimento para la intervención, solo que en cinco pacientes (1.3\%) hubo necesidad de liberar adherencias.

En los estudios se mencionan dos casos en los que se hizo resección parcial del hígado, el primero por hiperplasia ganglionar hepática y el segundo por sospecha de tumor en el lecho de la vesícula biliar.

Fue necesario convertir el procedimiento quirúrgico en siete casos (1.8\%), cinco a laparoscopia convencional por dificultad en la visualización del punto de seguridad. El sexto se convirtió a una colecistectomía por único puerto abdominal debido a la presencia de endometriosis pélvica. En el último caso se realizó cirugía abierta por adherencias severas y fibrosis de la vesícula biliar.

Las complicaciones no presentaron tasas altas. Solo en un artículo se informó complicación intraoperatoria $(0.2 \%)$ con perforación de la vejiga por mala técnica en la inserción del trocar vaginal. Las complicaciones postquirúrgicas fueron siete $(1.8 \%)$. Dos casos de hematuria que se resolvieron de forma espontánea en menos de doce horas. Una paciente con infección de la herida abdominal por conversión a cirugía abierta. Un caso de infección del tracto urinario y uno de infección fúngica vaginal. Los otros dos pacientes reingresaron a la tercera semana postoperatoria, con absceso en el fondo de saco de Douglas, ameritando drenaje por laparoscopia. No se reportaron sangrados ni lesiones del conducto biliar.

\section{DISCUSIÓN}

Los estudios revisados coinciden en afirmar que la CTV es un procedimiento quirúrgico seguro y clínicamente viable dentro de las cirugías mínimamente invasivas (14,16-18). También hay similares opiniones en cuanto a las ventajas que brinda este nuevo método operatorio, tales como: disminución del riesgo de formación de adherencias, reducción del riesgo de las hernias incisionales, menos infecciones de la herida, rápida recuperación y la más notoria es el resultado estético al no tener heridas visibles $(13,14,17,18,23)$.

Se han reportado preocupaciones respecto a su aplicabilidad y a posibles eventos adversos, tales como dolor en la pelvis, dispareunia, formación de fístulas biliares, abscesos en la bolsa de Douglas, punción de la vejiga urinaria, entre otros.

Por otro lado hay diferencias en los resultados dados por los estudios en cuanto a la duración de la estancia hospitalaria, el dolor postoperatorio y el consumo de analgésicos, algunos indican que estos no difieren en gran porcentaje de los resultados arrojados en la realización de la técnica laparoscópica convencional para la extracción de la vesícula biliar, otros por el contrario aseguran que estos tienden a ser mucho menores cuando se realiza CTV $(13,14,23)$.

El abordaje por colpotomía posterior a través del fornix, se dice que es más rápido, seguro y con menor índice de dolor, presentando pocas o nulas complicaciones en lo referente a: hematoma pélvico, infección del tracto urinario o infección de la herida quirúrgica (16-18).

Las lesiones de la vejiga urinaria pueden ser más comunes en un abordaje anterior por falta de compartimentación. En cuanto a la dispareunia y la disfunción sexual femenina, se cree que hay riesgo potencial debido a la cicatrización de la incisión de la pared vaginal $(14,16,23)$, pero los estudios existentes de los casos reportados no parecen ser una muestra significativa, los resultados estudiados son a corto plazo y con pocos períodos de seguimiento. No hay una respuesta aún a potenciales efectos postoperatorios en cuanto a fertilidad, sexualidad o problemas que puedan ser causados por el abordaje transvaginal.

En términos de seguridad, existe preocupación por la hermeticidad de los orificios por los cuales se llega a la cavidad abdominal, pues aunque existen sistemas para dicho procedimiento, tales como grapadoras, fórceps combinados con aguja de suturas, se cree que no son lo suficientemente eficaces y seguros para este cierre endoscópicamen- 
te. Pero hay quienes manifiestan que el cierre de la colpotomía es fácil y seguro para el paciente, sin complicaciones inmediatas ni tardías $(13,17)$.

En cuanto a la técnica quirúrgica algunos prefieren instrumentos flexibles, que le permitan llegar a la zona deseada, con mayor flexibilidad, y con menos riesgos de lesionar estructuras cercanas. A otros les resultan estos instrumentos poco adecuados, ya que les resulta difícil la manipulación y retracción de los órganos. En general CTV es una nueva técnica quirúrgica, que involucra avanzada tecnología para mejorar la tracción ejercida sobre la vesícula biliar y el hígado con el fin de exponer el pedículo hepático y lograr visión segura y permitir la disección precisa de las estructuras.

El tiempo operatorio promedio al emplear esta técnica, fue significativamente mayor que el ameritado para la colecistectomía laparoscópica, 162 minutos frente a $68 \mathrm{mi}-$ nutos en el segundo procedimiento. Se involucran en ello, a la falta de instrumentos endoscópicos adecuados y a la necesidad de curva de aprendizaje prolongada por los cirujanos $(18,23)$. No obstante, otro estudio ha reportado menor tiempo quirúrgico tanto con la CTV como con la colecistectomia laparoscópica convencional, 52 minutos frente a 35 minutos, respectivamente $p<0.001$ (18).

Se cuestiona la CTV por ser una técnica que requiere mayor tiempo quirúrgico y planea mayor grado de dificultad para el cirujano, solo para lograr mejores efectos cosméticos. Por otro lado, se ha pensado en el equilibrio costo-beneficio, pues para realizar este tipo de cirugías se necesita equipos costosos. Sin embargo, se cree que la solicitud en la práctica clínica puede aumentar una vez se haya desarrollado mejor técnica.

Aunque debe superar algunos obstáculos, como el acceso a cavidad abdominal sin complicaciones a través de la pared vaginal, el cierre seguro de dicha colpotomía, la prevención de infecciones y abscesos en la he- rida quirúrgica, la orientación espacial y la creación de herramientas adecuadas para su implementación.

Esta técnica es un método emergente que parece ser menos invasiva, mejor tolerada y más conservadora en cuanto a estética a diferencia de la colecistectomía laparoscópica. Lo que sitúa el mundo de la cirugía ad portas de un nuevo cambio cognoscitivo, el cual necesita ir de la mano con la ética médica, la economía y la seguridad en sentido de beneficio de los pacientes brindándoles mayor comodidad y una solución para su patología con menores perjuicios.

Cuando se introdujo la cirugía laparoscópica también se adentró en un cambio, y la historia demostró que pasó de ser un sueño descabellado al método de elección en la actualidad. Hoy la comunidad se enfrenta a uno nuevo y no es absurdo pensar en que esta se convertirá en la cirugía del futuro. NOTES podrá ser la mejor elección para abordar la vesícula biliar, si logra demostrar su superioridad sobre el abordaje quirúrgico que la antecedió.

Para lograrlo es necesaria la práctica de un mayor número de intervenciones y el registro de las mismas, que permita sentar bases sobre los criterios de inclusión, exclusión, complicaciones intra y postoperatorias, reducción del dolor y la necesidad de analgésicos después de la intervención quirúrgica.

\section{CONCLUSIÓN}

La CTV es una técnica quirúrgica que pudiese ofrecer buenos resultados operatorios, convirtiéndola en una técnica segura y factible cuando se realice en pacientes seleccionados. Se requieren más estudios.

CONFLICTOS DE INTERESES: ninguno que declarar.

FINANCIACIÓN: recursos propios de los autores.

\section{REFERENCIAS BIBLIOGRÁFICAS}

1. Litiasis biliar. Guías Clínicas. [Internet]. Fisterra; 2008 [Acceso 12 Marzo 2013]. Disponible en:http://www.fisterra.com/guias2/colelitiasis.asp 
2. Seguel G, Fres E, Fres M, Pinochet F, Espinosa R, Suárez A. Naturaleza de los pólipos de la vesícula biliar sometidos a colecistectomía. Rev. Chilena de Cirugía. 2007.59(3):208-11.

3. Andia M, Gederlini A, Ferreccio C. Cáncer de vesícula biliar: Tendencia y distribución del riesgo en Chile. Rev. Méd. Chile;135(5):565-74.

4. Martínez U, Arzabe J, Zamorano I, Palacios J, Pomier S, Soza Gonzalez PL. Incidencia de colelitiasis. [Internet] 2014. http://www.revistaciencias.com/publicaciones/EEkppZyZZALMCzVgzN.php

5. Ceramides L, Arteaga Y, Plaza T, Prieto Y, Hernández Z. Diagnóstico clínico y epidemiológico de la litiasis vesicular. Revisión bibliográfica. Rev. Ciencias Médicas. 2012;16(1):200-14.

6. Mendieta SR, Muñoz ML, Díaz G, Olguín P. Deficiencia con la edad del vaciamiento vesicular. Anales de Radiología. 2006;4:293-7.

7. González M, Bastidas BE, Panduro A. Factores de riesgo en la génesis de la litiasis vesicular. Investigación en Salud. 2005;VII:71-8.

8. Harris DP, Chateau IB, Miguel PJF. Litiasis biliar pediátrica en una población de alta prevalencia. Rev. Chil. Pediatr. 2007;78(5):511-8.

9. Rodríguez L, Sánchez A, Acosta L, Sosa R. Costes: colecistectomía laparoscópica frente a colecistectomía convencional. Rev Cubana Cir.2006;45(3):3-4.

10. Concepción de la Peña A, Soberbón I, Hernández Varea J, Cremata M. Resultados del tratamiento laparoscópico de la colecistitis aguda. 2009;48(2):0-0.

11. Varas L, Espinós CM, Bardají B. Cirugía endoscópica transluminal por orificios naturales (NOTES). Rev. Esp. Enferm. Dig. 2009;101(4):275-82.

12. González T, Rodríguez E, Nunez A. Consideraciones sobre la cirugía endoscópica transluminal a través de un orificio natural. Rev Cub Med Mil. 2011;40(3-4)285-94.

13. Cuadrado A, Noguera JF, Olea JM, Morales R, Dolz C, Lozano L, et al. Hybrid natural orifice transluminal endoscopic cholecystectomy:prospective human series. Surg Endosc. $2011 ; 25(1): 19-22$.

14. Van den Boezem PB, Velthuis S, Lourens HJ, Rahul AK, Samlal M A, Cuesta C S. Hybrid transvaginal cholecystectomy, clinical results and patient-reported outcomes of 50 consecutive cases. J Gastrointest Surg. 2013;17(5):907-12.

15. Mofid H, Emmermann A, Alm M, von Waldenfels HA, Felixmüller $C$, Zornig $C$. Is the tranvaginal route appropriate for intra-abdominal NOTES procedures? Experience and follow-up of 222 cases. Surg Endosc. 2013;27(8):2807-12.

16. Nijhawan S, Barajas JS, Majid S, Jacobsen GR, Sedrak MF, Sandler BJ, et al. NOTES transvaginal hybrid cholecystectomy: the United States human experience. Surg Endosc. 2013;27(2):514-7.

17. Noguera J, Dolz C, Cuadrado A, Olea J, Vilella A, Morales R. Hybrid transvaginal cholecystectomy, NOTES, and minilaparoscopy: analysis of a prospective clinical series. 2009; Surg Endosc. 2009;23(4):876-81.

18. Zornig C, Siemssen L, Emmermann A, Alm A, von Waldenfels HA, Felixmuller C, Mofid H. NOTES cholecystectomy: matched-pair analysis comparing transvaginal hybrid and conventional laparoscopic techniques in a series of 216 patients. Surg Endosc. 2011;25(6):1822-6.

19. Musquera M, Peri L, Izquierdo L, García E, Ribal MJ, Alcaraz A. Is LESS/NOTES surgery the new gold standard of minimally invasive surgery? Arch Esp Urol. 2013;66(1):161-7.

20. Domínguez L, Rivera A. Bermúdez C, Herrera W. Análisis de conversión durante colecistectomía laparoscópica a abierta en una cohorte prospectiva de 703 pacientes con colecistitis aguda. Rev. Cirugía Española. 2011;89(5):300-6.

21. Dávila F, Tsin DA. Cirugía por orificios naturales (NOTES y manos) ¿La tercera revolución quirúrgica? Rev. Asociación Mexicana de Cirugía Endoscopica.7(1-4):6-13.

22. Rosero AA, Crispín Valencia J, Muñoz A, Cadavid A. Coledocolitiasis. En: Asociación Colombiana de Cirugía. Guías de Manejo en Cirugía. Fecha de consulta: enero 5 de 2011. Disponible en: http://www.ascolcirugia.org/guiasCirugia/ coledocolitiasis.pdf

23. Mofid $\mathrm{H}$, Siemssen $\mathrm{L}$, Emmermann $M$, Alm HA, Waldenfels $\mathrm{V}$, Felixmüller $\mathrm{C}$. The transvaginal hybrid cholecystectomy: an enduring alternative to the laparoscopic cholecystectomy? Experience with 165 cases so far! Eur Surg. 2011;43(3):130-4.

Universidad de Cartagena
Fundada en 1827

REVISTA,CIENCIAS BIOMÉDICAS
Es el órgano de información científica de la Facultad de Medicina de la Universidad de Cartagena. Colombia.

Publique su trabajo en esta revista enviando su manuscrito a: revistacienciasbiomedicas@unicartagena.edu.co www.revistacienciasbiomedicas.com

Revista ciencias Biomédicas es una publicación independiente, imparcial, abierta, revisada por pares, de elevada visibilidad internacional, con circulación online e impresa. Publica artículos en todas las modalidades universalmente aceptadas en inglés y en español, de temas referentes con todas las ciencias biomédicas, incluyendo ámbitos clínicos, epidemiológicos o de estudios básicos.

El sistema de gestión de manuscritos es rápido y justo. Revista Ciencias Biomédicas está incluida en varias bases de datos latinoamericanas e internacionales.

Antes de enviar su manuscrito, revise las recomendaciones para los autores, presentes en: wWw.revistacienciasbiomedicas.com www.revistacienciasbiomedicas.com.co 